AJChE 2019, Vol. 19, No. 1, 25 - 37

\title{
Coal Fly Ash-based Geopolymer Spheres Coated with Amoxicillin and Nanosilver for Potential Antibacterial Applications
}

Brandon Cyril S. Lira ${ }^{1}$

Sophia Bianca A. Dellosa ${ }^{2}$

Casey Irene $L$. Toh ${ }^{2}$

Al Patrick A. Quintero ${ }^{2}$

Andre Leopold S. Nidoy ${ }^{2}$

Kimmie Dela Cerna ${ }^{3}$

Derrick Ethelbhert C. Yu ${ }^{1,4}$

Jose Isagani B. Janairo ${ }^{2,4,5}$

Michael Angelo B. Promentilla ${ }^{\star 3,4,6}$

${ }^{1}$ Chemistry Department, College of Science De La Salle University, 2401 Taft Avenue, Manila, Philippines

${ }^{2}$ Biology Department, College of Science De La Salle University, 2401 Taft Avenue, Manila, Philippines

${ }^{3}$ Chemical Engineering Department, Gokongwei College of Engineering De La Salle University, 2401 Taft Avenue, Manila, Philippines

${ }^{4}$ Materials Science and Nanotechnology Unit, Center for Natural Sciences and Environmental Research, De La Salle University, 2401 Taft Avenue, Manila, Philippines

${ }^{5}$ Biological Control Research Unit, Center for Natural Sciences and Environmental Research, De La Salle University, 2401 Taft Avenue, Manila, Philippines

${ }^{6}$ Center for Engineering and Sustainable Development Research, De La Salle University, 2401 Taft Avenue, Manila, Philippines

*e-mail: michael.promentilla@dlsu.edu.ph

Geopolymers are emerging "green" materials for its low embodied energy and carbon footprint, and its potential to valorize wastes, such as coal fly ash. It is an inorganic cementitious material formed from the polymerization of aluminosilicates in an activating solution such as that of alkali hydroxides or silicates. Their superior mechanical properties, including heat and fire resistance make them an excellent material for diverse applications. Recent studies have also exploited the tunable open porosity and adsorbing properties of geopolymers. Our work thus explores the potential of porous geopolymer spheres for antibacterial applications. These spheres were synthesized using coal fly ash as the geopolymer precursor and the porous surface is coated with either amoxicillin or silver nanoparticles (AgNPs) adsorbed in the matrix. For the AgNP geopolymer spheres, SEM images show spherical nanostructures when using ascorbic acid as a reducing agent, while spherical, cubical, and wire structures were observed when reduced using $\mathrm{NaBH}_{4}$. Indication from UV-Vis results also suggests the gradual release of both amoxicillin and AgNPs in the solution from the functionalized geopolymer spheres. Using E. Coli as the test organism for a modified disk diffusion assay, both showed zones of inhibition against the bacteria. Further tests on 
26 Coal Fly Ash-based Geopolymer Spheres Coated with Amoxicillin and Nanosilver for Potential Antibacterial Applications

antibacterial application of AgNP geopolymer spheres show their effectiveness to kill at least $95 \%$ of the $E$. coli in a water sample initially containing $10^{5} \mathrm{cfu} / \mathrm{mL}$ in just 30 minutes.

Keywords: Antibacterial, Geopolymer, Coal Fly Ash, Amoxicillin, Silver Nanoparticles

\section{INTRODUCTION}

Geopolymer has been recently recognized as an emerging alternative cementitious material to replace Portland cement for its low embodied energy and carbon footprint, and its potential to valorize waste such as coal fly ash, among others. Davidovits originally coined the term "geopolymer" in the 1970s to refer a class of material whose molecular structure consists of an amorphous to semi-crystalline inorganic network of mineral molecules (Davidovits 1991). They are formed from the polycondensation of aluminosilicates found for example in coal fly ash while reacting in an activating solution such as that of alkali hydroxides or silicates. Mechanical properties such as compressive and flexural strengths are at par with traditional cements, but have exceptional fire and chemical resistance. With these specific properties, numerous researches today focus on the use of geopolymers as a more environmentally friendly construction material (Singh et al. 2015). Moreover, recent studies on geopolymers have started to investigate its applications for other industries, particularly in refractory materials (Temuujin et al. 2011), in soil stabilization (Tigue et al. 2018), and lining for cement pipes to increase their durability (Chindaprasirt and Rattanasak 2016), among others.

Porous geopolymers are also being developed which are characterized by their large surface area and tunable pore sizes that can be utilized as cost-effective replacements for different media since they can be used as adsorbents, catalysts, sensors, filters, and thermal insulators (Asl et al. 2018). In addition, a study by Armayani et al. in 2017 determined the antibacterial property of a silver nanoparticle modified geopolymer composite to inhibit growth of certain types of bacteria that is applied as an antibacterial coating material for walls and floors. Another related study by De Muynck et al. in 2008 utilized geopolymer mortars by making them antimicrobial by adding triclosan and benzalkonium chloride. Results show that the geopolymer mortar with triclosan inhibits the growth of Escherichia coli, Listeria monocytogenes, Salmonella enterica, and Staphylococcus aureus, where large zones of inhibition were observed, indicating that antimicrobial concrete mixtures are feasible and can be used to improve the hygienic conditions of various environments.

In this study, porous geopolymer spheres based from coal fly ash and waste pen shells (De La Cerna et al. 2018) were enhanced with antibacterial properties by coating the porous surface with amoxicillin 
Brandon Cyril S. Lira, Sophia Bianca A. Dellosa, Casey Irene L. Toh, Al Patrick A. Quintero, Andre Leopold S. Nidoy, Kimmie Dela Cerna, Derrick Ethelbhert C. Yu, Jose Isagani B. Janairo, Michael Angelo B. Promentilla 27

and silver nanoparticles (AgNPs). Morphological characterizations were also done using scanning electron microscopy (SEM). In addition, the dissociation of these antimicrobial geopolymers in solution were measured using UV-Vis spectrophotometry. Antibacterial activity test of these geopolymer spheres were also done using $E$. coli as the indicator.

\section{MATERIALS AND METHODS}

\section{Preparation of Geopolymer Spheres}

Geopolymer spheres were synthesized using a modified method described in Tang et al. 2015. This study used coal fly ash and calcined waste pen shells as the main raw materials. Foaming agents were added as well to increase the porosity of the spheres. The fly ash and the calcined shells were first combined at a total weight of $80 \mathrm{~g}$ to act as a geopolymer precursor and form the spherical beads. An amount of $7.5 \mathrm{~g}$ of a $12 \mathrm{M} \mathrm{NaOH}$ solution was added with $30.0 \mathrm{~g}$ of WGS to form the alkali activator solution. The solid precursor was then added to the alkali activator and stirred by hand for 1 minute. $1.7 \mathrm{~mL}$ of water was also added, then hand-stirring was done for another 2 minutes. Finally, varying masses of a $50 \%$ $\mathrm{H}_{2} \mathrm{O}_{2}$ solution was dropped in, ranging from $0.15 \%$ to $0.35 \%$ of the mass of the geopolymer. This was stirred thoroughly for another 2 minutes. The resulting paste was brown and had a thick consistency.

The geopolymer paste was then loaded into a syringe and injected dropwise into a hot PEG-600 solution at $90^{\circ} \mathrm{C}$. The dropwise addition of the paste, coupled with the high temperature of the medium, allows the paste to rapidly solidify into 4-5 $\mathrm{mm}$ beads. The formed spheres were left in the PEG-600 for about 20 minutes in order to fully harden; they were then collected, rinsed thoroughly, and left to cure in a laboratory oven at $90^{\circ} \mathrm{C}$ for another 24 hours. Fig. 1 shows the synthesized geopolymer spheres

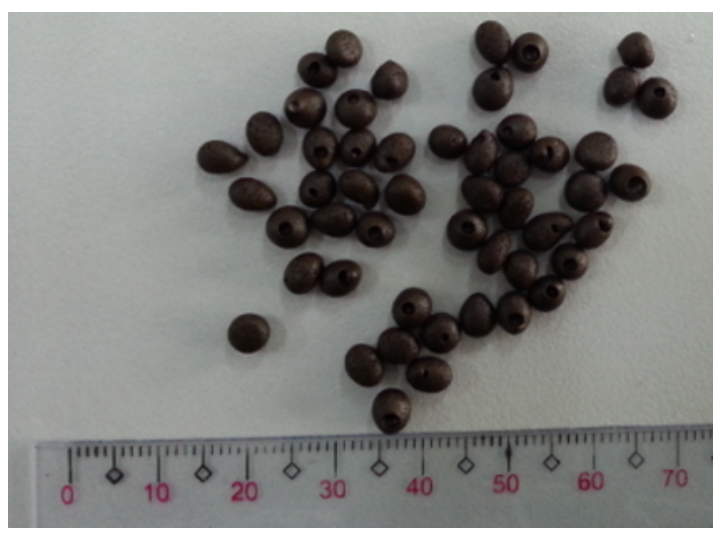

Fig. 1: Geopolymer spheres from coal fly ash and calcined waste pen shells

\section{Coating of Amoxicillin in Geopolymer Spheres}

In a $15 \mathrm{~mL}$ centrifuge tube, a solution of $1 \mathrm{gram}$ of amoxicillin dissolved in $5 \mathrm{~mL}$ of distilled water was prepared. The geopolymer spheres were immersed in this solution for 24 hours. They were then removed from the solution and were allowed to air dry at room temperature for another 24 hours.

\section{Coating of Geopolymer Spheres with AgNPs}

This method of coating geopolymer spheres with AgNPs was based on the study by Banne et al. 2017 and was modified to use geopolymer spheres as adsorbent media. Three sets of 
28 Coal Fly Ash-based Geopolymer Spheres Coated with Amoxicillin and Nanosilver for Potential Antibacterial Applications

geopolymer spheres were rinsed thoroughly with distilled water and were left to dry in open air at room temperature. Next, the geopolymer spheres were submerged in $1 \mathrm{~mL}$ of $1 \mathrm{M}$ $\mathrm{AgNO}_{3}$ inside a microcentrifuge tube for 1 hour. Afterwards, the $\mathrm{AgNO}_{3}$ solution was discarded from the spheres, and another 1 $\mathrm{mL}$ of $1 \mathrm{M} \mathrm{AgNO}_{3}$ was added and left to submerge for another hour. Then, the geopolymer spheres were transferred in a petri dish to air dry.

To produce AgNPs, in situ reduction of $\mathrm{AgNO}_{3}$ inside the treated geopolymer spheres was done by submerging one set in $1 \mathrm{~mL}$ of $1 \mathrm{M} \mathrm{NaBH}_{4}$, and another set in in $1 \mathrm{~mL}$ of $1 \mathrm{M}$ ascorbic acid, both for 1 hour. To confirm the presence of AgNPs in the reducing agent treated geopolymer spheres, surface morphology was determined using scanning electron microscopy and was compared to the third set in which no reducing agent was added which corresponds to the control sample.

\section{Dissociation Efficacy of the Geopolymer Spheres via UV-Vis Spectrophotometry}

\section{A. Amoxicillin Coated Geopolymer Spheres}

The ability of the loaded spheres to function as antibacterial agents highly depends on its dissociation activity. Thus, it is essential to quantitatively determine the amount of amoxicillin dissociated from the loaded spheres. This was done by determining the lambda max of the antibiotic solution using a Hitachi U-2900 UV-Vis spectrophotometer in which dilutions of the mixture were scanned 800 $200 \mathrm{~nm}$. With this, the absorbance values of the amoxicillin dilutions were used for the construction of the calibration curve.

In a Falcon tube, the amoxicillin loaded geopolymer spheres were immersed in $5 \mathrm{~mL}$ of distilled water together with a magnetic stirrer. The contents of the tube were then mixed for three minutes. Then, a $3 \mathrm{~mL}$ portion of this mixture was transferred to a quartz cuvette to measure its absorbance at the 500-200 $\mathrm{nm}$ range, with distilled water as the reference. After the analysis, the contents of the cuvette were put back in the Falcon tube and continued mixing for another 3 minutes before measuring the absorbance again. This procedure was repeated until a total of 30 minutes of mixing time was achieved.

\section{B. AgNP Functionalized Geopolymer Spheres}

The same procedure was done to the geopolymer spheres with adsorbed AgNPs, except a single geopolymer sphere was immersed in $4 \mathrm{~mL}$ of distilled water and the contents of the tube were shaken mildly at a constant motion. Every 3 minutes for 30 minutes, the absorbance of the solution was recorded, with wavelengths ranging from $600 \mathrm{~nm}$ to 250 $\mathrm{nm}$.

\section{Antimicrobial Test of the Geopolymer Spheres}

\section{A. Modified Standard Disk Diffusion Technique}

Their bactericidal effect against $E$. coli using a modified standard disk diffusion technique was used in the following geopolymer sphere samples: 
Brandon Cyril S. Lira, Sophia Bianca A. Dellosa, Casey Irene L. Toh, Al Patrick A. Quintero, Andre Leopold S. Nidoy, Kimmie Dela Cerna, Derrick Ethelbhert C. Yu, Jose Isagani B. Janairo, Michael Angelo B. Promentilla 29

$\mathrm{NaBH}_{4}$ reduced and ascorbic acid reduced AgNPs, with $\mathrm{AgNO}_{3}$ only, and the amoxicillin loaded samples.

Prior to antibacterial testing, Mueller-Hinton agar (MHA) was prepared according to the standard protocol of Clinical \& Laboratory Standards Institute (CLSI) in which $38 \mathrm{~g}$ of agar were suspended per $1000 \mathrm{~mL}$ of distilled water. The mixture of agar and distilled water was heated to ensure homogeneity before being autoclaved. After sterilization, the agar mixture was cooled to $45-50^{\circ} \mathrm{C}$, mixed well, and was poured into sterile petri dishes.

The bacteria, E. coli, were first transferred into a cooled flask containing the MHA before being poured into respective petri dishes. Afterwards, instead of using standard paper disks. For each plate, four geopolymer spheres were submerged in the MHA before it completely solidifies. The plates contain approximately $1.5 \times 10^{8}$ suspension of $E$. coli. The amount of $E$. coli was determined using $0.5 \mathrm{McF}$ arland Standard. The zones of inhibition the geopolymer spheres showed after 24 hours of incubation in $37^{\circ} \mathrm{C}$ were then recorded using a Vernier caliper.

B. Antimicrobial efficiency of AgNPgeopolymer spheres in E-coli contaminated water

A $10^{5} \mathrm{cfu} / \mathrm{mL}$ inoculum was first prepared using a culture of $E$. coli made 24 hours prior to the experiment. The $30 \mathrm{~mL}$ of this inoculum was placed in a beaker containing $\mathrm{NaBH}_{4}$ reduced AgNPgeopolymer spheres. The beaker was then agitated in an orbital shaker set at 300 rpm. Exactly $1.5 \mathrm{~mL}$ of $5 \% \mathrm{w} / \mathrm{w}$ solution of sodium thiosulfate was added 30 minutes after. This immediately halts the antimicrobial activity of the silver ions, ensuring a constant contact time of 30 minutes was done for all of the samples. Then, $1 \mathrm{~mL}$ of the liquid from each sample and their serial dilutions (up to $10^{-3}$ ) were plated in agar and left to incubate at $37^{\circ} \mathrm{C}$ for 24 hours. Plate counting was then done to determine the final concentration of $E$. coli in the water. Note that this plate counting technique was also done to determine the initial $E$. coli count of the water.

\section{RESULTS AND DISCUSSION}

Coating of Geopolymer Spheres with AgNPs

Geopolymer spheres coated with AgNPs were synthesized in situ by the reduction of $\mathrm{AgNO}_{3}$ with either $\mathrm{NaBH}_{4}$ or ascorbic acid. Investigation of the geopolymer spheres through SEM shows distinct differences in their morphologies. The untreated geopolymer spheres, as shown in Fig. 2, are immersed only in distilled water. This control sample shows smooth areas that make up the surface of the neat geopolymer.

The geopolymer spheres with AgNPs from the reduction by ascorbic acid produced spherical silver nanostructures (Fig. 3a) with uneven densities of the AgNPs surrounding the geopolymer, while the ones reduced by $\mathrm{NaBH}_{4}$ also produced spherical shapes, alongside with uneven cubic nanostructures, and silver nanowires (Fig. 3b). 
30 Coal Fly Ash-based Geopolymer Spheres Coated with Amoxicillin and Nanosilver for Potential Antibacterial Applications

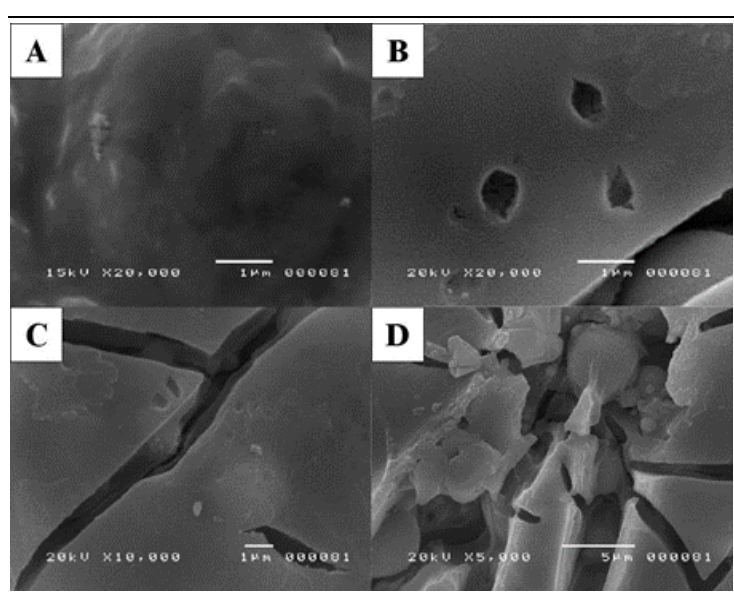

Fig. 2: SEM Micrograph of geopolymer spheres immersed only in distilled water.

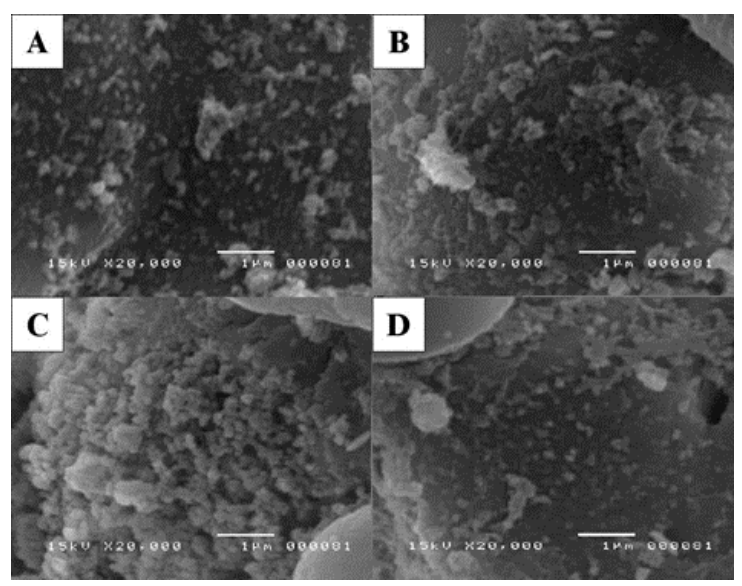

(a)

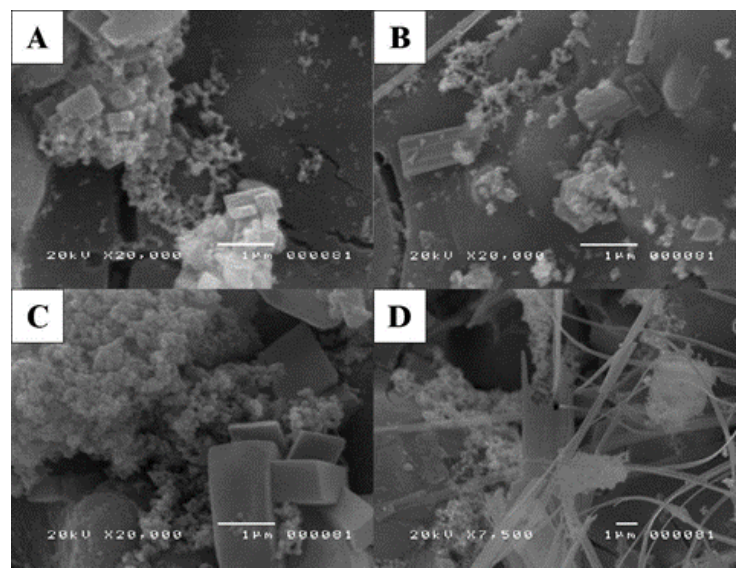

(b)

Fig. 3: SEM micrographs of geopolymer spheres with AgNPs prepared by reducing $1 \mathrm{M} \mathrm{AgNO}_{3}$ (a) with $1 \mathrm{M}$ ascorbic acid (b) with $1 \mathrm{M} \mathrm{NaBH}_{4}$.
The spherical nature and the varying densities and sizes of the AgNPs formed by reduction of ascorbic acid was suspected to be due to the absence of a stabilizing agent during the reduction process. This was supported from a study by Al-Thabaiti et al. in 2013, in which the role of stabilizers in the formation of nanostructures are important. It was found out that the presence of stabilizers or surfactants, such as cetyltrimethylammonium bromide (CTAB), leads to an increased formation of monodisperse spherical nanostructures.

On the other hand, $\mathrm{NaBH}_{4}$, generated AgNPs with varying morphologies on the surface of the geopolymer spheres. Since $\mathrm{NaBH} 4$ is the stronger reducing agent, its reaction to form AgNPs are rapid as compared to reduction by ascorbic acid. This rapid reduction does not allow proper nucleation of the AgNPs and instead forms various sizes and morphologies (Khodashenas \& Ghorbani 2015).

Another possibility to consider is that the geopolymers contain other reagents used for its synthesis. Polyethylene glycol is used to synthesize geopolymer spheres. A study by Cong et al. noted that silver nanowires may be synthesized by reducing $\mathrm{AgNO}_{3}$ in polyethylene glycol (PEG) with poly (vinyl pyrrolidone) (PVP) as a capping agent (Cong et al. 2012). Also, a similar study was introduced by Sun and Xia in 2002, wherein they synthesized cubic AgNPs by reducing $\mathrm{AgNO}_{3}$ with ethylene glycol in the presence of PVP. It is possible that remnants of the PEG-600 solution may have remained in the geopolymer due to 
Brandon Cyril S. Lira, Sophia Bianca A. Dellosa, Casey Irene L. Toh, Al Patrick A. Quintero, Andre Leopold S. Nidoy, Kimmie Dela Cerna, Derrick Ethelbhert C. Yu, Jose Isagani B. Janairo, Michael Angelo B. Promentilla 31

the sphere's porosity, even though they were thoroughly washed, thus affecting the development of the AgNPs, and eventually creating silver nanowires and cubic AgNPs in the process.

\section{Dissociation Efficacy of the Geopolymer Spheres via UV-Vis Spectrophotometry}

For the geopolymer spheres to become effective antibacterial carriers, it is important that the functional materials would adhere to its structure longer. Since its porosity allows components to incorporate themselves within the geopolymer, it may also readily allow the antibacterial agent to leach or dissociate from it.

\section{A. Amoxicillin Coated Geopolymer Spheres}

The lambda max of the antibiotic mixture was determined to be at $350 \mathrm{~nm}$. Thus, absorbance values of the amoxicillin dilutions at $350 \mathrm{~nm}$ were obtained to get the calibration curve as shown in Eq. 1 and Fig. 4.

$y=0.0752 x-0.0042$

Using the calibration curve, the concentrations of the diffused amoxicillin per time was obtained. Since the concentration is directly proportional to the absorbance values, this means that as the geopolymer spheres spend more time mixing in the solution, the more antibiotic is dissociated over time as denoted by its increasing trend (Fig. 5). Furthermore, the dispersion of the drug is gradual; the antibiotic did not completely dissociate all at once, as the spheres were immersed in water. The chemical structure of the amoxicillin may have contributed to this phenomenon as it contains different functional groups which are capable of hydrogen bonding to the geopolymer network, hindering the full release of the drug. The steady release of the drug over time the idea that the geopolymer sphere is a possible drug carrier.

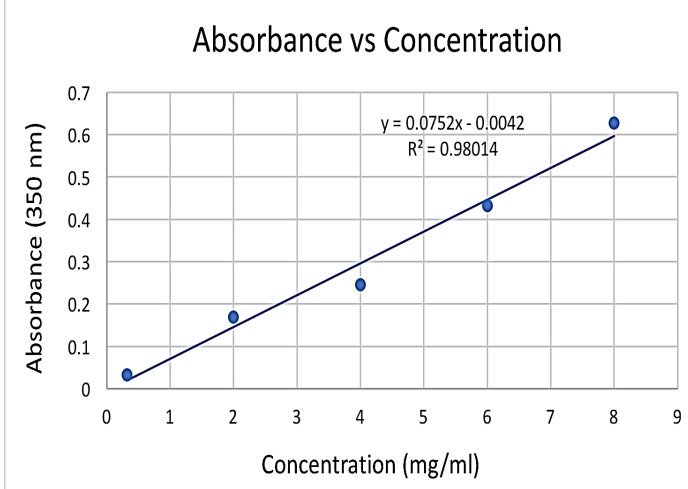

Fig. 4: Absorbance versus concentration calibration curve of the diluted amoxicillin solutions read at 350 $\mathrm{nm}$.

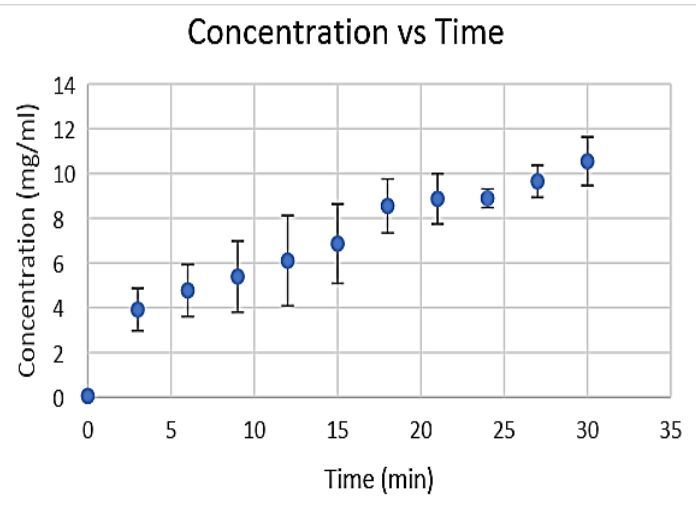

Fig. 5: Graph of average concentration values of dissociated amoxicillin over the course of 30 minutes.

\section{B. AgNP Coated Geopolymer Spheres}

To test the geopolymer spheres' efficacy to contain the AgNPs, the absorbance of distilled water immersed 
32 Coal Fly Ash-based Geopolymer Spheres Coated with Amoxicillin and Nanosilver for Potential Antibacterial Applications

with AgNP geopolymer spheres reduced with $\mathrm{NaBH}_{4}$ over 30 minutes was measured.

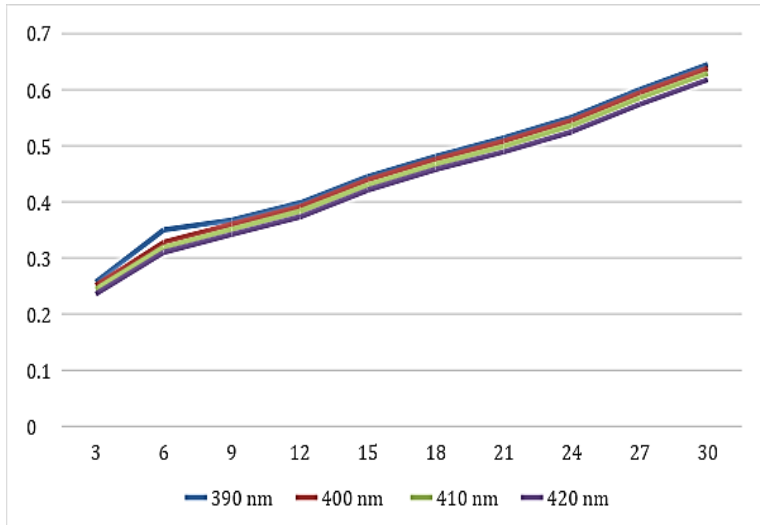

Fig. 6: Absorbance of distilled water with AgNP geopolymer reduced by $\mathrm{NaBH}_{4}$ from $390-420 \mathrm{~nm}$ for 30 minutes

The solution was scanned in the 600-250 $\mathrm{nm}$ range and comparison of values shows a significant increase in absorbance after 30 minutes. The absorbance peak, set at $250 \mathrm{~nm}$, increased from 0.377 to 0.95 after half an hour. According to a study by Tulve et al. in 2015, AgNPs have a peak absorbance in the wavelength range of 390 to $420 \mathrm{~nm}$.

It is seen in Fig. 6 that the absorbance of the distilled water solution exposed to the functionalized geopolymer spheres has a linear relationship with as time increases. Furthermore, the dispersion of the AgNPs is gradual as the length of mixing time increases; the AgNPs did not completely dissociate all at once, as the spheres were immersed in water.

\section{Preliminary Antimicrobial Testing}

\section{A. Geopolymers Spheres with AgNPs}

To determine the antibacterial efficacy of the geopolymer spheres impregnated with AgNPs, the zone of inhibition (ZOI) against $E$. coli was determined using an antibacterial susceptibility test. AgNP geopolymer spheres reduced using $\mathrm{NaBH}_{4}$ displayed significant ZOls from 7-10 mm, showing that these geopolymers were successful in inhibiting the growth of bacteria (Fig. 6a). On the other hand, the synthesized AgNP geopolymer spheres reduced using ascorbic acid did not show any ZOls (Fig. 6b).

A known cause of AgNPs' antibacterial activity is its ability to increase the bacterial cell's permeability. Due to this, the cell fails to regulate the transport of substances between its membrane, eventually leading to its death. These nanoparticles accumulate in the cell membrane and form pits in the cell wall (Stoimenov et al. 2002, Sondi \& SalopekSondi 2004, Li et al. 2010). Also, the silver particles disrupt the replication of bacterial DNA by the diffusion of $\mathrm{Ag}$ through the cell membrane which allows it to react with and inactivate thiol containing proteins. This then allows $\mathrm{Ag}$ to bind to DNA, thus inhibiting replication (Feng et al. 2000). Further studies show that treatments of AgNPs induced a direct effect over the cell's mitochondrial function associated with the generation of reactive oxygen species, caspase-3 activation and DNA fragmentation based on their size (Sriram 2012).

The size of the AgNPs also greatly affect its cytotoxicity. Smaller particles are shown to have more efficient antibacterial activity as compared to larger particles (Baker et al. 2005). The synthesis of AgNPs 
Brandon Cyril S. Lira, Sophia Bianca A. Dellosa, Casey Irene L. Toh, Al Patrick A. Quintero, Andre Leopold S. Nidoy, Kimmie Dela Cerna, Derrick Ethelbhert C. Yu, Jose Isagani B. Janairo, Michael Angelo B. Promentilla 33

by reduction with $\mathrm{NaBH}_{4}$ generally yields smaller particles (Pyatenko et al. 2007) which is mainly due to the reducing power of $\mathrm{NaBH}_{4}$. The reduction by the weaker reducing agent, ascorbic acid, yielded clumped and agglomerated structures (Fig. 2a) which ultimately affected its antibacterial activity.

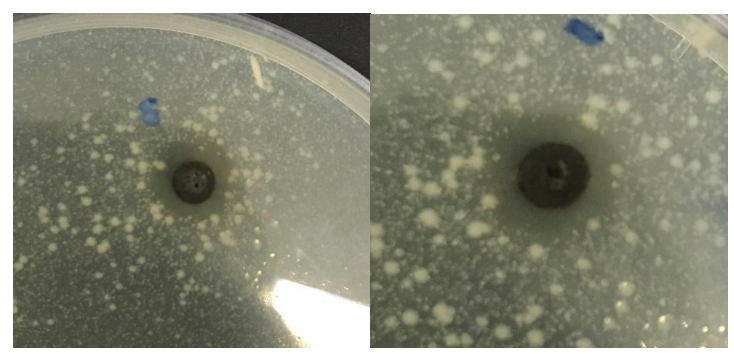

(a)

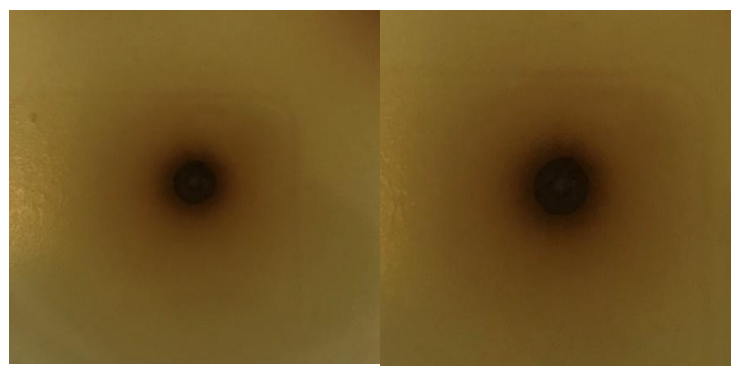

(b)

Fig. 7: Zones of inhibition of $E$. coli exposed to AgNP adsorbed geopolymer reduced by (a) $\mathrm{NaBH}_{4}$ (b) ascorbic acid

It was also found out that while reduction by ascorbic acid indeed synthesized AgNPs, it is inefficient in keeping them inside the geopolymers as shown in Fig. 6b. It showed no ZOls, but instead a discoloration of the agar medium due to the leached Ag. Another trial, wherein the geopolymers were only soaked in $\mathrm{AgNO}_{3}$ and then tested against E. coli, formed uneven ZOIs (Fig. 7) and a brown discoloration was also detected, primarily due to $\mathrm{AgNO}_{3}$.
One-way analysis of variance was then used to determine if there is a statistically significant difference between the ZOls yielded by AgNP geopolymer reduced using $\mathrm{NaBH}_{4}$, ascorbic acid, and control geopolymers spheres. Since the $p$ value $(1.23 \mathrm{e}-19)$ is lower than the significance level of 0.05 , the null hypothesis is rejected. Thus, there is a significant difference between the ZOls of the $\mathrm{NaBH}_{4}$ reduced AgNP treated geopolymer compared to those untreated samples.

In addition, the $\mathrm{NaBH}_{4}$ reduced AgNP-geopolymer spheres were evaluated for antimicrobial efficiency in a $E$. coli contaminated water using Eq. 2.

$$
A E=\frac{\text { initial count }- \text { final count }}{\text { initial count }} \times 100 \%
$$

Table 1 summarizes the results from this preliminary anti-microbial test after the spheres are in contact with the $\mathrm{E}$ cili contaminated water for 30 minutes. It is evident from the results that majority of the trials were successful in eliminating at least $95 \%$ of the $E$. coli in the water sample.

Table 1. Antimicrobial Efficiencies (AE) of AgNP-geopolymer spheres

\begin{tabular}{cc}
\hline Trial & AE (\%) \\
\hline 1 & 95.46 \\
\hline 2 & 97.97 \\
\hline 3 & 98.88 \\
4 & 98.65 \\
\hline 5 & 99.94 \\
\hline
\end{tabular}


34 Coal Fly Ash-based Geopolymer Spheres Coated with Amoxicillin and Nanosilver for Potential Antibacterial Applications

\section{B. Geopolymer Spheres with Amoxicillin}

Antibacterial testing is also a means to determine the drug carrying efficiency of the geopolymer spheres. For the spheres to be a candidate as a potential carrier, no adverse reaction between the drug and the geopolymer should occur.

The geopolymers were autoclaved after immersion in antibiotic solution and prior to exposure to $E$. coli in MuellerHinton agar; $1.0 \mathrm{~g} / 5 \mathrm{~mL}$ was the dosage used for the antibiotic solution to assure immediate results. After 24 hours of incubation, no ZOIs were observed and bacterial growth were present around the spheres, and is possibly due to the evaporation of the antibiotic during the process of sterilization through autoclaving.

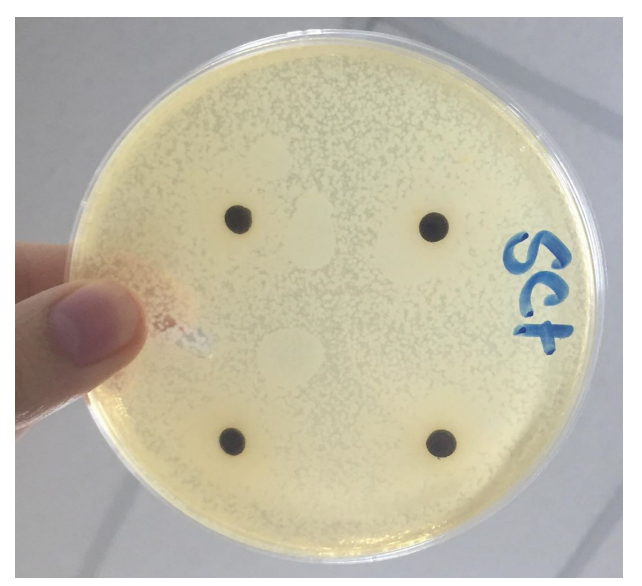

Fig. 8: Zone of inhibition of the amoxicillin loaded and autoclaved geopolymer spheres in E. coli.

Autoclaving resulted in the degradation of amoxicillin from the geopolymer spheres. Thus, by omitting this step, the antibiotic should remain to be loaded into the geopolymer. All the geopolymer spheres were able to exhibit significant ZOls at around $19-20 \mathrm{~mm}$ (Fig. 9).

Through a one-way analysis of variance, it was observed that the $p$-value (3.217 e-11) is lower than the significance level of 0.05 . Thus, the null hypothesis is rejected, meaning that there is a significant difference between the treated and the untreated spheres.

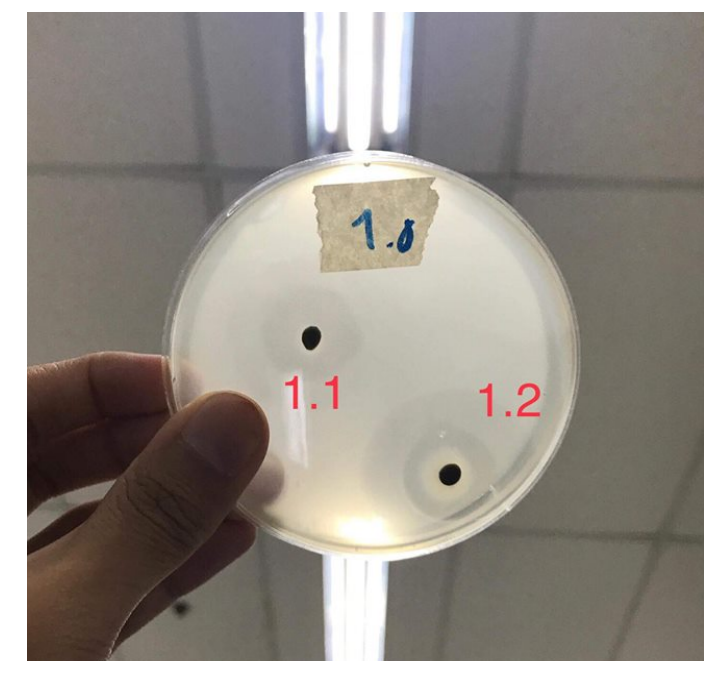

Fig. 9: Zone of inhibition of the amoxicillin loaded and unautoclaved geopolymer spheres in $E$. coli.

After incubation, bacterial growth was observed near the ZOls (Zone of Inhibitions). This can be accounted to the amoxicillin being completely dissociated. If amoxicillin were not completely dissociated, no bacterial growth should have occurred. With this it can be inferred that the geopolymer spheres possess the ability to effectively carry and fully deliver the drug loaded unto it, allowing them to be promising drug carriers.

\section{CONCLUSIONS}

Coal fly ash based geopolymer 
Brandon Cyril S. Lira, Sophia Bianca A. Dellosa, Casey Irene L. Toh, Al Patrick A. Quintero, Andre Leopold S. Nidoy, Kimmie Dela Cerna, Derrick Ethelbhert C. Yu, Jose Isagani B. Janairo, Michael Angelo B. Promentilla 35

spheres were coated or loaded in their porous surface with AgNPs and amoxicillin to exhibit antibacterial properties which are determined via susceptibility tests against $E$. Coli. This study determined that the geopolymer spheres with amoxicillin displayed a larger ZOI as compared to the spheres with AgNPs reduced by $\mathrm{NaBH}_{4}$. Meanwhile, AgNPs reduced by ascorbic acid showed no ZOls due to its weak reduction strength and have agglomerated AgNPs as shown in SEM. Spheres with AgNPs reduced by $\mathrm{NaBH}_{4}$ were tested for antimicrobial efficiency and shows to kill at least $95 \%$ of the $E$. coli in a water sample initially containing $10^{5}$ $\mathrm{cfu} / \mathrm{mL}$ in just 30 minutes. Note that these only aim to serve as preliminary studies and future work will be done to further investigate the potency of their antibacterial effect. Nevertheless, both applications show gradual dissociation of the added functionalities in the geopolymer spheres as determined by UV-Vis spectroscopy. This shows also that the coal fly ash based geopolymer spheres can be used as a carrier medium for AgNPs and amoxicillin for potential antimicrobial applications.

\section{ACKNOWLEDGMENT}

The authors would like to acknowledge the financial support from the Department of Science and Technology (Philippines) through their Engineering Research and Development for Technology (ERDT) scholarship program and Philippine Council for Industry, Energy, and Emerging
Technology Research and Development Advanced Device and Materials Testing Laboratory (PCIEERD-ADMATEL) grant, and URCO Research Project.

\section{REFERENCES}

1. Ahmaruzzaman, M. (2010). "A review on the utilization of fly ash". Progress in Energy and Combustion Science, 36(3), 327-363.

2. Armayani., M., Akbar Pratama, M., \& Subaer. (2017). "The Properties of Nano Silver (Ag)-Geopolymer as Antibacterial Composite for Functional Surface Materials". MATEC Web of Conferences, 97, 01010.

3. Baker, C., Pradhan, A., Pakstis, L., Pochan, D., Shah, SI. (2005). "Synthesis and Antibacterial Properties of Silver Nanoparticles". J Nanosci Nanotechnol. 5(2):244-249.

4. Chindaprasirt, P., \& Rattanasak, U. (2016). "Improvement of durability of cement pipe with high calcium fly ash geopolymer covering". Construction and Building Materials, 112, 956-961.

5. Cong, F-Z., Wei, H., Tian, X-R., Xu, H-X. (2012). "A facile synthesis of branched silver nanowire structures and its applications in surface-enhanced Raman scattering". Front Phys. 7(5):521-526.

6. Davidovits, J. (1991). "Geopolymers". Journal of Thermal Analysis, 37(8), 1633-1656.

7. Dela Cerna, K. Janairo, J.I., Promentilla M.A., (2018). "Development of nanosilver-coated geopolymer beads (AgGP) from fly ash and baluko shells 
36 Coal Fly Ash-based Geopolymer Spheres Coated with Amoxicillin and Nanosilver for Potential Antibacterial Applications

for antimicrobial applications". MATEC

Web of Conferences 268, 05003.

8. De Muynck, W., Belie, ND, Verstraete, W. (2008). "Antimicrobial mortar surfaces for the improvement of hygienic conditions". Journal of Applied Microbiology. 108: 62-72.

9. Department of Energy 2012. List of Existing Power Plants-Luzon. Available from

https://www.doe.gov.ph/doe_files/pdf/ 01_Energy_Situationer/2012-Power-

Plants-Luzon.pdf

10. Feng, Q.L., Wu J., Chen, G.Q., Cui, F.Z., Kim, T.N., Kim, J.O. (2000). "A mechanistic study of the antibacterial effect of silver ions on Escherichia coli and Staphylococcus aureus". Journal of Biomedical Materials Research. 52(4):662-668.

11. Geopolymer Institute. What is a geopolymer? Introduction. https://www.geopolymer.org/science/i ntroduction/ (accessed Oct 10, 2018).

12. Khodashenas, B., Ghorbani, H.R. (2015. "Synthesis of silver nanoparticles with different shapes". Arab J Chem.

13. Li W-R, Xie X-B, Shi Q-S, Zeng H-Y, Ou-Yang Y-S, Chen Y-B. (2009). "Antibacterial activity and mechanism of silver nanoparticles on Escherichia coli". Applied Microbiology and Biotechnology. 85(4):1115-1122.

14. Pyatenko A, Yamaguchi $M$, Suzuki $M$. (2007). "Synthesis of spherical silver nanoparticles with controllable sizes in aqueous solutions". J. Phys Chem C. 111(22):7910-7917.

15. Singh, B.; Ishwarya, G.; Gupta, M.; Bhattacharyya, S. (2015). "Geopolymer concrete: A review of some recent developments". Constr. Build. Mater. 2015, 85, 78-90.

16. Sondi, I., Salopek-Sondi, B. (2004). "Silver nanoparticles as antimicrobial agent: A case study on E. coli as a model for Gram-negative bacteria". J. Colloid Interface Sci. 275(1):177-182.

17. Sriram, M.I., Kalishwaralal, K., Barathmanikanth, S., Gurunathani, S. (2012). "Size-based cytotoxicity of silver nanoparticles in bovine retinal endothelial cells". Nanosci Methods. 1(1):56-77.

18. Stoimenov, P.K., Klinger, R.L., Marchin, G.L., Klabunde, K.J. (2002). "Metal oxide nanoparticles as bactericidal agents". Langmuir. 18(17):6679-6686.

19. Sun, Y., Xia, Y., Melillo, J.M., Bowles, F.P., Melillo, J.M., Steudler, P.A., Rayment, M., Trumbore, S.E., Amundson, R., Bergh, J., et al. (2002). "Shape-controlled synthesis of gold and silver nanoparticles". Science. 298(5601):2176-9.

20. Tang, Q., Ge, Y.-Y., Wang, K.-T., He, Y., \& Cui, X.-M. (2015). "Preparation of porous P-type zeolite spheres with suspension solidification method". Materials Letters, 161, 558-560.

21. Temuujin, J., Rickard, W., Lee, M., \& van Riessen, A. (2011). "Preparation and thermal properties of fire resistant metakaolin-based geopolymer-type coatings". Journal of Non-Crystalline Solids, 357(5), 1399-1404.

22. Tigue, A.A.S., Malenab, R.A.J., Dungca, J.R., Yu, D.E.C., Promentilla, M.A.B. (2018). "Chemical stability and leaching behavior of one-part geopolymer from soil and coal fly ash mixtures". Minerals, 8 (9), 411. 
Brandon Cyril S. Lira, Sophia Bianca A. Dellosa, Casey Irene L. Toh, Al Patrick A. Quintero, Andre Leopold S. Nidoy, Kimmie Dela Cerna, Derrick Ethelbhert C. Yu, Jose Isagani B. Janairo, Michael Angelo B. Promentilla 37

23. Tulve, N.S., Stefaniak, A.B., Vance, M.E., Rogers, K., Mwilu, S., Lebouf, R.F., Schwegler-berry, D., Willis, R., Thomas, T. (2015). "Characterization of silver nanoparticles in selected consumer products and its relevance for predicting children's potential exposures". 218(3):345-357.

24. Williams, D. (2015). Philippines set for 23 new coal-fired power plants. Power Engineering International. Available from

https://www.powerengineeringint.com /articles/2015/06/23-new-coal-firedpower-plants-for-philippines.ht 



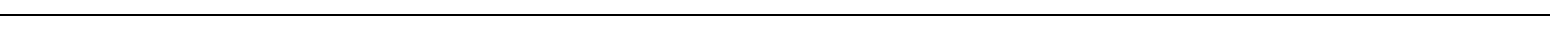

